\title{
The Management of the Atlanto-Occipital and Atlanto-Axial Joint Pain
}

\author{
Bentley A. Ogoke, MD
}

Headaches secondary to atlanto-occipital and atlanto-axial joints are often associated with suboccipital pain and aggravated by lateral rotation of the cervical spine and/or flexion or extension of the cervical spine. They are frequently under-diagnosed using traditional radiological evaluation including plain $\mathrm{X}$-rays, computerized tomographic scan, and magnetic resonance imaging.

While the natural history of most acute atlanto-occipital and atlanto-axial joint inflammation is resolution over a 2to-4-week period with conservative management, however, when chronicity sets in, resolution is difficult to achieve using conservative measures, and interventional pain management procedures may be indicated.

This review will describe the anatomy of the upper cervical spine, clinical presentation of atlanto-occipital and atlanto-axial joint involvement, and various modalities of treatment, including conservative and interventional modalities.

Keywords: Atlanto-occipital joint, atlanto-axial joint, chronic pain, nerve blocks
Pain arising from inflammation of the upper cervical spine joints, namely, the atlanto-occipital and atlanto-axial joints, respectively, typically presents with occipital headaches, which are often associated with suboccipital pain and aggravated by lateral rotation of the cervical spine and/or flexion or extension of the cervical spine (1-4). Very often, these may be related to trauma, e.g., head injury or whiplash, or arthritis. They are frequently underdiagnosed since there is typically no X-ray (as in open-mount view of the cervical spine) or computerized tomographic (CT) scanning or magnetic resonance imaging (MRI) wherein gross structural abnormality (destruction) is evident. However, the clinical presentation is often reliable and reproducible in the proper clinical scenario. By far, the most common etiology in the pain of atlanto-occipital and atlanto-axial joint inflammation is acceleration-deceleration injury, blunt head trauma, and osteoarthritis of the upper cervical spine.

The natural history of most acute atlanto-occipital and atlanto-axial joint inflammation is resolution over a 2-to-

From Pioneer Valley Pain Management and Palliative Medicine Center, Inc., Springfield, Massachusetts. Dr Ogoke is medical director of Pioneer Valley Pain Management and Palliative Medicine Center, Inc. Address correspondence: Bentley Ogoke, MD, 125 Liberty Street Suite 100, Springfield, Massachusetts 01103 4-week period with conservative management. However, when chronicity sets in, resolution is difficult to achieve using conservative measures, and interventional pain management procedure(s) may be indicated.

\section{ANATOMY OF THE UPPER CERVICAL SPINE}

To understand invasive and noninvasive treatment of this headache syndrome, it is necessary to outline the gross anatomy of the skeletal and vascular portion of the upper cervical spine and the base of the skull. The atlanto-occipital joint is the articulation of the superior articular facet of the $\mathrm{C} 1$ vertebrae (atlas) and the occiput. These two joints can be seen as modified facet (zygapophysial) joints. The atlanto-occipital joint straddles the spinal cord and the foramen magnum on either side and is $V$-shaped, slanting caudally and medially. The joint has a capsule and is a synovial joint with a healthy thickness of cartilage on the skull base as well as on the articular surface of the atlas vertebrae. The vertebral artery runs medially and diagonally (to enter the skull's foramen magnum) and is often located overlying the medial one-third of the joint. Other surrounding structures include the carotid artery and jugular veins anterolaterally. The atlanto-axial joint is located below and constitutes the $\mathrm{C} 1-2$ joint. The atlanto-axial slopes caudally and laterally and has the same articular characteristics as the atlanto-occipital in that both are synovial joints. The vertebral artery runs on the lateral one-third of the joint dorsally and then goes through 
the $\mathrm{C} 1$ vertebrae (atlas) superiorly to pass into the foramen magnum medially. Inflammatory or arthritic processes can occur at either or both joints. The atlas and axis do not have intervertebral foramina. The $\mathrm{C} 1$ and $\mathrm{C} 2$ nerves, which are primarily sensory, travel through muscle and fascia to the occipital area as the greater occipital nerves and the lesser occipital nerves (5).

Radiologic diagnosis of the inflammatory process has a high false-negative result since pain onset often precedes any noticeable structural (destructive) abnormality of the joints of the cervical spine involved (atlanto-occipital and atlanto-axial joints). Typically, X-ray of the cervical spine, MRI of the cervical spine, and CT scan are negative. Clinically, history and examination are diagnostic; while MRI of the cervical spine and skull (brain) may be done to rule out other causes of neck pain and occipital headaches. It is important to note that occipital headaches can arise from injuries affecting the disc of the $\mathrm{C} 2-3$, as well as its facet joints and the atlantoaxial and atlantooccipital joints. The lower cervical spine vertebral levels do not cause occipital headaches. However, the coexistence of muscle spasm either primarily or secondarily initiated by lower cervical spine area pathology can also lead to occipital headaches.

The atlanto-occipital joint permits flexion and extension passive range of motion (ROM)of about 10 degrees and 25 , degrees respectively; while the atlanto-axial joint permits lateral rotation of about 70 degrees on either side, with the movement occurring around the odontoid process. The atlanto-axial also allows isolated flexion and extension of five degrees and ten degrees, respectively, in and of itself.

\section{CLINICAL PRESENTATION}

The atlanto-occipital and atlanto-axial joints are often evaluated and treated at the same time. The joints when inflamed are known to present with occipital, suboccipital and neck pain. Headache is the prominent feature of this pathology; it is constant and varies in intensity, and may be associated with "tightness" in the neck and suboccipital region. The natural history of this problem passes through an acute and a chronic phase, with the acute phase being by far the most prevalent; although the disease tends to stop at this stage, but it may still not resolve and progress to a chronic phase.

The acute phase is managed conservatively, combining oral, non-steroidal antiinflammatory drugs and other ad- juvant medications, including muscle relaxants and tricyclic antidepressants, as well as physical therapy to achieve resolution. The chronic phase often requires invasive pain management blocks before progress can be made to achieve resolution. Episodes of nausea may occur rarely; blurry vision and tinnitus can also occur with acute exacerbation and may be unilateral. Involvement of the lesser occipital nerves and severe tenderness and guarding elicited on palpation are often associated with blurry vision. Insomnia is a frequent finding as well. Neck pain and reduced cervical spine range of motion are common. Rarely, the maxillary branch of the trigeminal nerve may also be involved, with symptoms referred to the preauricular region.

Other associated symptoms will depend on the etiology of the inflammatory process in the atlanto-occipital and atlanto-axial joints. When patients present with whiplash syndrome, they may have other symptoms of postconcussion syndrome or aggravated cervical spondylosis superimposed on the symptoms of the atlanto-occipital and atlanto-axial inflammation. These may vary in severity. Dizziness, lightheadedness, concentration difficulties, episodes of mild disorientation and difficulty with memory and recall may be part of the presenting complaint. These are more common with motor-vehicle accidents, falls, and sports-related injuries to the head. Degenerative osteoarthritis etiology tends to produce neck pain and occipital headaches primarily.

Atlanto-occipital and atlanto-axial joints tend to elicit aggravation and tenderness with flexion-extension ROM and lateral ROM, respectively, as outlined in Tables 1-3.

Dynamics of isolated ROM of the atlanto-occipital joint

Table 1. Clinical presentation of atlantooccipital and atlanto-axial joint disease

\begin{tabular}{ll}
\hline- & Occipital and suboccipital headaches \\
Reduced range of motion of C-spine and head \\
Neck pain \\
- & Insomnea (infrequent) \\
Pain and/or restriction with nodding move- \\
ments (atlanto-occipital joint disease) \\
Referred pain with the preauricular region \\
(trigemino-cervical nucleus) \\
Blurry vision \\
Tinnitus
\end{tabular}


Table 2. Mechanisms of atlanto-occipital joint pain

Inflammation of joints (degenerative, traumatic,
infectious, rheumatoid, etc.)
Synovial joint adhesions
A combination of inflammation, joint adhesions
and loss of or reduced cartilage thickness of joint
in advanced cases
Capsular irritation and inflammation leading to
sensitivity to stretch (nodding)
Referred pain to the occipital and suboccipital
region (headaches)
Referred pain to the temporal region that can
extend to the preauricular area to the temporo-
mandibular-joint region

show 25 degrees of extension and 10 degrees of flexion. Palpation of the paravertebral muscles of the upper cervical spine tends to elicit tenderness with guarding and trigger points may also be present. Radiographic studies are often negative, including X-ray of the cervical spine with the odontoid view (open mouth), CT of the upper cervical spine, and the cervical spine and the brain on MRI. They are often performed to rule out other etiologies. Arthrography of the joints is usually benign. The diagnosis of atlanto-occipital and atlanto-axial joint pain is usually a clinical diagnosis and derived primarily from history and physical examination.

Table 3. Mechanisms of atlanto-axial joint pain

- Inflammation (degenerative, traumatic, infectious, rheumatoid)

- Intra-articular chondral adhesions

- Chronic degenerative loss of intra-articular cartilage in combination with the above

- $\quad$ Capsular inflammation and irritation due to increase of sensitivity with stretch

- $\quad$ Pain referred mostly to the suboccipital region

- $\quad$ Possibility of occipital headache (and pain)

- $\quad$ Referred pain through $\mathrm{C} 1$ to $\mathrm{C} 3$ dorsal rami that converges with the trigeminal afferent via trigeminocervical nucleus; may cause maxillary area $\mathrm{V} 2$ pain in the preauricular region

- $\quad$ Periarticular muscle spasm (hypertonus and hypertonicity)

- $\quad$ Possibility of 60 degrees to 90 degrees of horizontal rotation around odontoid

- Possibility of isolated 10 degrees of extension and 5 degrees of flexion of atlanto-axial joint
Table 4. Differential diagnoses of atlantooccipital and atlanto-axial pain

\begin{tabular}{llll}
\hline Vascular & CNS & Intracranial & Musculoskeletal \\
\hline $\begin{array}{l}\text { Vertebral } \\
\text { artery } \\
\text { aneurysm }\end{array}$ & $\begin{array}{l}\text { Inflammation } \\
\text { of dura by } \\
\text { blood or } \\
\text { infection }\end{array}$ & Tumor & $\begin{array}{l}\text { Discopathy of upper } \\
\text { cervical spine, e.g., } \\
\text { C2-3 degenerative } \\
\text { disc disease and C2- } \\
\text { 3 herniation }\end{array}$ \\
$\begin{array}{llll}\text { Temporal } \\
\text { arteritis }\end{array}$ & $\begin{array}{l}\text { C1-3 nerve } \\
\text { root irritation }\end{array}$ & AV & malformation \\
& $\begin{array}{l}\text { Atlanto-occipital and } \\
\text { atlanto-axial } \\
\text { inflammatory } \\
\text { arthropathy (facet) }\end{array}$ \\
$\begin{array}{l}\text { Basilar } \\
\text { artery spasm } \\
\text { (migraine) }\end{array}$ & $\begin{array}{lll}\text { Arnold-Chiari } \\
\text { malformation }\end{array}$ & Hemmorhage & $\begin{array}{l}\text { Tender muscle } \\
\text { ligaments }\end{array}$ \\
& & & C2-3 facet \\
& & arthropathy
\end{tabular}

The differential diagnosis (Table 4) of atlanto-occipital and atlanto-axial joint pain includes vertebral artery aneurysm, which shares the same nerve supply as the joints; as well as basilar artery spasm, which may produce migrainous attacks, and meningeal inflammation by blood or infection, in that region which also depends on the same nerve supply. The $\mathrm{C} 1$ to $\mathrm{C} 3$ nerve root irritation can also produce similar symptoms. The Arnold-Chiari malformation, tumor, arterial venous malformation and upper cervical discopathy and C2-3 facet arthropathy may also mimic atlanto-occipital and atlantoaxial joint pain.

\section{TREATMENT}

The treatment of atlanto-occipital and atlanto-axial joint pain is not always invasive, as previously mentioned. In the acute phase an initial trial of conservative therapy is indicated. This includes the use of oral medications including high-dose non-steroidal antiinflammatory drugs, muscle relaxants, tricyclic antidepressants, oral prednisone tapered or short-term narcotic use as an adjunct to control pain. In addition, physical therapy should be used, including modalities such as hot pack, traction, electrical stimulation, massage, ROM exercises, joint mobilization, ultrasound and craniosacral therapy.

Acupuncture and chiropractic manipulation also have a place in the conservative management of these patients.

The invasive management of atlanto-occipital and atlantoaxial joint pain that responds poorly to conservative easures often provides quick and lasting relief or resolu- 
tion by use of atlanto-occipital and atlanto-axial joint injections employing local anesthetics and depo-steroids under fluoroscopic guidance. Indications for atlanto-occipital and atlanto-axial joint injections are listed in Table 5. It is typical to "cover" both joints during this treatment. There are a number of techniques and positions for both the patient and fluoroscopy (C-arm) machine described in the literature. Patients may require either one or two injections. The patient, following informed consent, is placed in a prone position with a pillow under the chest and a folded blanket to support the forehead with only slight neck flexion or none, depending on pre-existing axial spine contour. All procedures are done under strict aseptic conditions. The beam of the $\mathrm{C}$-arm is directed in the axis or direction of the occiput and the tip of the jaw at the midline with $\mathrm{C}$-arm adjustment to expose the atlanto-occipital and atlanto-axial joints. The final view is obtained by instructing the patient to open his/her mouth. This view provides a modified open-mouth view of the cervical spine, which provides clear visualization of the joints.

Following skin infiltration with $1 \%$ lidocaine, a $25-\mathrm{G} x$ 3.5 " needle(s) is placed via an introducer, depending on the size of the patient, into the atlanto-occipital and atlanto-axial joint as desired under fluoroscopic guidance. The lateral half of the atlanto-occipital joint is used, while the medial half of the atlanto-axial joint is pierced by the needle. A contrast injection may be necessary in difficult

\section{Table 5. Indications for atlanto-axial and atlanto-occipital injections \\ 1. Degenerative disorders of atlanto-occipital and atlanto-axial joints \\ 2. Traumatic inflammation of atlanto-occipital and atlanto-axial joints \\ 3. Persistent occipital neuralgia poorly responsive to occipital nerve blocks \\ 4. Diffuse cervical facet arthropathy with persis- tent upper-neck (occipital) pain and headaches \\ 5. Whiplash syndrome with prominent intractable occipital headaches \\ 6. Upper-level cervical spondylosis (osteoarthri- tis) with poor response to serial epidural ste- roid injections, associated with persistent oc- cipital head pain \\ 7. Pain of osteoarthritis involving the upper cervi- cal spine joints (facet) and the odontoid \\ 8. Ankylosing spondylitis involving atlanto-occipi- tal joints}

cases to confirm needle placement through the arthrography, followed by injection of 1.5 to $2 \mathrm{cc}$ of $0.125 \%$ bupivacaine and $20 \mathrm{mg}$ of Depo-Medrol ${ }^{\circledR}$ to each side. Lower concentrations of bupivacaine have also been used when the patient has a fragile cardiovascular status, or when concern about leak is an issue due to multiple punctures of the capsule of the atlanto-occipital and/or atlantoaxial joints concerned. It is important to recognize that the therapeutic effect is obtained eventually from the injected depo-steroid, and unnecessary use of high-concentration local anesthetics in this delicate region increases the risk of the procedure without added therapeutic benefits.

A distinct "pop" is usually felt on entering the joint capsule or space. The patient is observed supine for at least 40 to 45 minutes before discharge, with monitoring of vital signs.

Complications are uncommon in experienced hands; but side effects of dizziness, nausea, ataxia, metallic taste in the mouth, and vomiting do occur.

\section{COMPLICATIONS}

The following are complications and side effects of atlantoaxial and atlanto-occipital injections:

1. Infection- Aseptic conditions should prevail during this procedure considering the proximity of the joints at the base of the brain and increased vascularity that may lead to infectious arthritis or meningitis;

2. Seizure- This can result from intravascular injection of at least $1 \mathrm{cc}$ of local anesthetic or less, the most implicated artery being the vertebral artery;

3. Total spinal block- This can result when there is an accidental intrathecal injection;

4. Nausea and vomiting- these are common when high-volume injections are done and during the peri-injection period;

5. Spinal headaches (post-dural puncture headaches);

6. Respiratory arrest;

7. Hypotension- (frequently secondary to local anesthetic effects through an accidental epidural injection of high concentration of local anesthetic);

8. Direct cord injury and paralysis- (rare);

9. Pneumoencephalitis;

10. Injury to Arnold-Chiari malformation and other 
cerebellar tonsillar herniation abnormalities;

11. Episodes of dizziness and ataxia immediately post injection- (common); and

12. Other local anesthetic toxicity, including a metallic taste in the mouth and perioral numbness.

Epidural/spinal injection, as well as intravascular injection, into the vertebral artery are real risks. The latter can lead to seizure even with less than $1 \mathrm{cc}$ of local anesthetic injected, depending on concentration, e.g., $0.5 \%$ bupivacaine or $2 \%$ lidocaine.

The risk/benefit ratio for this procedure warrants that more effort be made to diagnose and treat this headache syndrome, as relief is often quick and long lasting and frequently leads to resolution. Following atlanto-occipital and atlantoaxial joint injection when other associated problems have been diagnosed or are present, it might be necessary to address such simultaneous diagnoses as, for example, postconcussion syndrome, which may also cause headache; as well as treatment of lower cervical and mid cervical spine strain, which may produce muscle spasms in the paravertebral muscles of the cervical spine and tends also to perpetuate the headache. Other possible causes include cervical herniation or an aggravated facet arthropathy within other portions of the cervical spine as well, including the $\mathrm{C} 2-3$ level.

\section{CONCLUSION}

It is known that atlanto-occipital and atlanto-axial joints of the upper cervical spine are capable of generating head and neck pain. The natural history of the involvement of these joints shows the resolution over a 2-to-4-week period with conservative management. However, in patients in whom chronicity sets in, resolution is difficult to achieve using conservative measures. Hence, interventional pain management procedures with injections of the joints or nerve blocks are indicated. Invasive modalities are associated with risks and complications. However, individualization of patient treatment with proper technique and meticulous attention to detail will not only avoid such complications but also will be successful in managing these patients with chronic headache.

\section{REFERENCES}

1. Dreyfuss P, Michaelsen M, Fletcher D. Atlanto-occipital and atlanto-axial joint pain patterns. Spine 1994; 19:1125-1131.

2. Busch E, Wilson PR. Atlanto-occipital and atlantoaxial injections in the treatment of headache and neck pain. Reg Anesth 1989; 14:45-47.

3. McCormick CC. Arthrography of the atlanto-axial (C1-C2) joints: Technique and results. J Intervention Radiol 1987; 2:9-11.

4. Dreyfuss P, Roberts J, Dreyer S et al. Atlanto-occipital joint pain: A report of three cases and description of an intra-articular joint block technique. Reg Anesth 1994; 19:344-351.

5. Bogduk N. Clinical anatomy of the cervical dorsal rami. Spine 1982; 7:319-330. 